\title{
In Vitro Evaluation of Marginal Sealing Ability of Bulk Fill Flowable Composite Resins
}

\author{
Ruchi Gupta ${ }^{1}$,Anil K Tomer ${ }^{2}$,Anamika Kumari \\ ${ }^{I}$ Professor, Dept Of Conservative Dentistry And Endodontics, Divyajyoti College Of Dental Sciences And \\ Research, Modinagar, Ghaziabad \\ ${ }^{2}$ Professor And Head, Dept Of Conservative Dentistry And Endodontics, Divyajyoti College Of Dental Sciences \\ And Research, Modinagar, Ghaziabad \\ ${ }^{3}$ PG Student, Dept Of Conservative Dentistry And Endodontics, Divyajyoti College Of Dental Sciences And \\ Research, Modinagar, Ghaziabad
}

\begin{abstract}
:
Aim: The purpose of this study is to analyse marginal sealing ability of bulk fill flowable composite resins. Materials and Methods: A standard class $V$ cavity $(3 \mathrm{~mm}$ mesiodistal width, $3 \mathrm{~mm}$ occlusogingival height, and $1.5 \mathrm{~mm}$ axial depth) will be prepared at the cementoenamel junction (CEJ) on the facial surface of eighty human teeth with a $0.5 \mathrm{~mm}$ wide $45^{\circ}$ beveled enamel margin and a $90^{\circ}$ cervical margin; the gingival margin will be placed $1.0 \mathrm{~mm}$ below the CEJ. The specimens were restored with different flowable composite resins:Surefil SDR flow (Dentsply, York, PA, USA), TetricEvo flow bulk fill (Ivoclarvivadent), Filtek bulk fill (3M ESPE), Xtra base (voco). The specimens will be immersed in a $0.5 \%$ basic fuchsin dye solution for 24 hours and sectioned. The degree of microleakage was checked under stereomicroscope. . The data was statistically analysed by Kruskall-Wallis test.
\end{abstract}

Results: There was non-significant difference in marginal sealing ability of different bulkfill flowable composite resins at enamel and dentin.

Conclusion: The marginal sealing ability of bulkfill composite resin was not influenced by the type of substrate or flowable composite resin.

Keywords: Bulkfill, flowable, composite resin, microleakage

In Vitro Evaluation Of Marginal Sealing Ability Of Bulk Fill Flowable Composite Resins

\section{Introduction}

Composite resins are considered material of choice in restorative dentistry because of the increasing demand for high-quality esthetic results in everyday practice. They have been widely used in the restoration of posterior teeth due to increasing demand for esthetics as well as dramatic improvement in newer generation bonding agents and resin composite formulations. The currently available nanocomposite restorative systems are suitable for both anterior and posterior restorations as they provide adequate resistance in high stress bearing areas and superior esthetics than microhybrids. ${ }^{1-4}$ Nevertheless, despite the continuous evolution of these resins, problems such as polymerization shrinkage and marginal microleakage still occur. Stresses from shrinkage create forces that compete with the adhesive bond, and this may disrupt the bond to cavity walls, which is still one of the main causes of marginal failure and subsequent microleakage especially in dentin margins of restorations. Furthermore, with high-viscosity composite resin, it is difficult to obtain perfect adaptation to the internal cavity surface and proper marginal seal of the cavity. Many attempts have been made to decrease microleakage of adhesive restorations, such as development of new resin monomers and filler systems, incremental filling technique, type of light source, light and curing mode and changes in C-factor and direction of polymerization shrinkage. The restorative techniques that reduce the level of stress due to resin composite polymerization shrinkage have been suggested. ${ }^{5-8}$

Adhesion has been demonstrated to be either micro-mechanical, due to the penetration of the bonding resin inside the microscopic and submicroscopic surface imperfections of the enamel \& dentin and chemical, due to the partial dentin demineralization that leave a substantial amount of hydroxyapatite crystals around the collagen fibrils. The ability of the adhesive resin to infiltrate enamel and dentin is related to the surface wettability and it depends on the amount of surface free energy of dental substrate, which is directly proportional to the level of mineralization and indirectly proportional to the percentage of organic tissue. This clearly explains the higher predictability of adhesion to enamel compared with dentin. ${ }^{9}$ One possible solution to the weaker seal on dentin is the use of more adaptable flowable resins on enamel-free margins.

Flowable composite resins are widely used in clinical practice and are the most common resin materials that are recommended for restoring these lesions instead of conventional resin composites. Bulk Fill flowable resins 
with improved mechanical and chemical characteristics have recently been introduced. ${ }^{3}$ Flowable resin composites are low-viscosity materials with the reduced percentage of inorganic filler particles $(44-55 \%$ in volume) and higher amount of resinous components. ${ }^{10-12}$ Consequently, the polymerization process leads to an important volumetric contraction, but with minimal stress contraction. According to Hooke's Law, stress is determined by volumetric shrinkage and the elastic modulus of the material. Flowable composites, with their low elastic modulus compete with stress development, potentially helping to maintain the marginal seal of the restoration. Moreover, flowable composites are readily workable and adaptable to cavity walls and their use can reduce marginal defects in restorations. These materials have good aesthetic properties. ${ }^{13-15}$

To the best of our knowledge, there is no reported study assessing the microleakage of these new flowable resins on both enamel and dentin. The aim of this in vitro study is to assess the marginal sealing ability of bulkfill flowable composite resins on both enamel and dentin.

\section{Material And Methods}

A total of 80 freshly extracted human premolars without decay, cracks or previous restorations will be selected, thoroughly cleaned, and stored in physiological saline solution at room temperature. Each tooth underwent scaling and root planing with an ultrasonic device to remove residual organic tissue. Then, the teeth were immersed in $2.6 \%$ sodium hypochlorite solution and rinsed with running water for 10 min.Teeth were stored in distilled water at room temperature until used for the experiment.

A standard class $\mathrm{V}$ cavity ( $3 \mathrm{~mm}$ mesiodistal width, $3 \mathrm{~mm}$ occlusogingival height, and $1.5 \mathrm{~mm}$ axial depth) was prepared at the cementoenamel junction (CEJ) on the facial surface of each tooth with a $0.5 \mathrm{~mm}$ wide $45^{\circ}$ beveled enamel margin and a $90^{\circ}$ cervical margin; the gingival margin will be placed $1.0 \mathrm{~mm}$ below the CEJ. The preparation will be performed with a straight fissure diamond bur (MANI Ltd., Utsunomiya, Japan) in a water-cooled high-speed handpiece by one operator. The bur will be replaced after every five preparations.

The specimens will be randomly divided into four groups $(n=20)$ and restored with different flowable composite resins:

Groups I Surefil SDR flow (Dentsply, York, PA, USA)

Group II TetricEvo flow bulk fill (Ivoclarvivadent)

Group III Filtekbulkfill (3M ESPE)

Groups IV X-tra base (voco)

The adhesive system was applied in all the samples according to the manufacturers instructions. All samples were restored using bulk technique and subsequently polymerized. After $24 \mathrm{~h}$, the restorations were finished with fine-grit diamond bur and polished. The prepared samples were subjected to thermocycling in water baths for 500 times between 5 and 55 degrees with a dwell time of 30 seconds in each bath and a transfer time of 30 seconds to simulate the oral conditions.

The samples were stored in distilled water at $37^{\circ} \mathrm{C}$ for 7 days. Five teeth serve as the positive control in which no restoration was done to ensure the dye penetration. Five teeth serve as the negative control which were covered by sticky wax all around to ensure no penetration of the dye. All the experimental teeth were completely sealed with two layers of nail polish, leaving 1-mm window around the cavity margins. They were immersed in a $0.5 \%$ basic fuchsin dye solution for 24 hours. The specimens were rinsed in running water and then dried. The teeth were sectioned faciolingually with a low speed diamond saw under water spray.

The dye penetration depth along the cavity wall was measured with a stereomicroscope at $20 \times$ magnification. Two independent precalibrated investigators blindly scored dye penetration (microleakage) along the toothrestoration interface and consensus was forced when disagreements occurred. The microleakage score was recorded separately for both occlusal and cervical margins on a nonparametric ordinal scale from 0 to 3 as

$\mathbf{0}=$ no dye penetration

$\mathbf{1}=$ dye penetration involving less than half the occlusal/gingival wall

$\mathbf{2}=$ dye penetration involving more than half the occlusal/gingival wall

$\mathbf{3}=$ dye penetration involving the axial wall. The data will be statistically analyzed.

The scoring criteria for the microleakage assessment were followed according to Sadeghi et al. ${ }^{2}$ The same procedure was followed for all the remaining samples. Data was collected, tabulated and sent for statistical analysis.

\section{Results}

The frequencies of microleakage scores obtained from the experimental groups for the enamel and dentin margins are presented in Table 1. The mannwhitney and Kruskall-Wallis analysis of variance revealed non significant microleakage differences among the four groups at enamel and dentin margins $(P>0.05)$. 
Resin-based composite restorations bonded with TetricEvoflow bulkfill showed least microleakage, and the highest microleakage values were displayed by Filtekbulkfill. All of the systems appeared to be incapable of sealing the restoration and, thus, preventing microleakage through the enamel dentin margins.

\begin{tabular}{|c|c|c|c|}
\hline Groups* & Mean & Std. Deviation & n \\
\hline I - E & 1.26 & 0.733 & 20 \\
\hline I - D & 2.50 & 0.513 & 20 \\
\hline II- E & 1.20 & 0.696 & 20 \\
\hline II- D & 2.43 & 0.598 & 20 \\
\hline III-E & 1.21 & 0.713 & 20 \\
\hline III-D & 2.50 & 0.513 & 20 \\
\hline IV -E & 1.23 & 0.718 & 20 \\
\hline IV -D & 2.47 & 0.616 & 20 \\
\hline
\end{tabular}

Table 1: Mean values of degree of microleakage at enamel and dentin margins using different flowable bulkfill composite resins

\section{Discussion}

One of the most important aims of cavity restorations is to establish predictable marginal seal in order to prevent microleakage and its clinical consequences such as marginal discrepancies, marginal staining, recurrent caries, sensitivity and pain. The bulkfill flowable composite resin possess a lower modulus of elasticity, as well as lower levels of polymerization stress in comparison to traditional flowable composite, without compromising on depth of cure. ${ }^{16-18}$ The material is marketed as a resin composite for bulk application in direct composite resin restorations. The rapid development of dental materials, and the time needed for in vivo and in vitro studies has meant that the effects of bulk fill composite resin on marginal micro-leakage have not received sufficient attention. Therefore, this study aimed to investigate the effect of a new-generation bulkfillflowable composite resin on micro-leakage in Class $\mathrm{V}$ composite restorations. The null hypothesis of the study was that micro-leakage would not be affected by types of flowable composite.

Dental restorations are subjected to constant and extreme changes in the oral environment, with large fluctuations in temperature and $\mathrm{pH}$. Restorative materials are constantly exposed to thermal variations during the intake of food and fluids at various temperatures. Thus, thermocycling is an important procedure for testing the sealing ability of restorative material. Thermally induced stresses, which may lead to gap formation and microleakage, result from the mismatch between the coefficients of thermal expansion for the restorative material and the natural tooth structure. The samples were subjected to thermocycling in order to replicate the intraoral environment. ${ }^{19}$

Dye penetration is an established in vitro method for investigating marginal leakage along toothrestoration interfaces and is generally assessed after cutting the teeth in the longitudinal direction. Various tracer dyes are available for microleakage studies and there appears to be no significant difference in tracer penetration among fuchsin, silver nitrate and methylene blue. $0.5 \%$ basic fuschin is one of the most common tracers and can be used at different concentrations. SEM requires that the sample be subjected to extensive chemical preparations before the scanning process and this may lead to alteration or even destruction of the interfacial zones, and even underestimation of the actual thickness of the hybrid layer. For this reason, immersion in $0.5 \%$ basic fuschin was used in the present study and this immersion time was suitable for marking marginal gaps.

The degree of microleakage was calculated at the tooth-restoration interface for both the enamel and dentin, which were filled with four different bulkfill flowable composite materials: Surefil SDR, Tetric Evoflow bulkfill, Filtek bulkfill \& X-tra base. Because of the study setup, flowable resins were placed in the cavities without covering it with resin composite. In fact, in order to evaluate the efficacy of the tested materials to avoid the marginal microleakage on both enamel and dentin, it was necessary to seal the interfaces exclusively with the material object of study.

In the current study, we examined the microleakage of different types of bulkfill flowable composite resins at enamel and dentin margins. None of the interfaces showed an absence of infiltration, although the degree of infiltration differed in relation to the substrate and the material. The dye penetration was not significantly affected by the marginal substrate and its interaction with the resin material. The percentage of infiltration on enamel was same as that on dentin. This can be explained by the lower stress due to low elastic modulus and lower wettability of bulkfill flowable composite. This finding is in contrary to the previous studies regarding adhesion on enamel and dentin. ${ }^{4}$ The presence of less dye penetration and better marginal seal on enamel in these studies is related to the low organic content of enamel compared with dentin; dentin has a complex structure rich in organic molecules, making adhesion to dentin more variable and difficult to achieve. However on enamel margins, flowable composite showed low percentage of infiltration, confirming that the quality of adhesion on enamel is able to overcome curing shrinkage, regardless of the volumetric shrinkage of the resinous material employed ${ }^{20}$ Furthermore, flowable composites can be readily inserted into small cavities 
and are expected to adapt better to the internal cavity wall than other restorative composites, which are more viscous. These features of flowable composites can account for our findings of their superior behavior. Bulk-fill composite materials evaluated in the present study seem to meet satisfactorily the requirements of this type of materials in terms of marginal adaptation. Bulk-fill composites are more translucent than other restorations, which allow the light to get to much deeper layers. The content of photoinitiators of polymerization and stress inhibitors determines the optimal marginal seal of these composites.

In the present study the most favorable results were obtained when the application of the material took place using TetricEvoflow bulkfill. The degree of fluidity when applying the composite material influences the marginal adaptation; increased fluidity of the composite makes it adhere better to the walls of the cavity. Moreover the chemistry of the bulkfill flowable composite resin affects the polymerization shrinkage leading to difference in marginal sealing ability. Thus, the type of substrate and the material employed are the key parameters to be considered when restoring a tooth.

\section{Conclusion}

Within the limitations of the present study, it can be concluded that the marginal sealing ability of bulkfill composite resin was not influenced by the type of flowable composite resin or the type of substate i.e. enamel or dentin used for bonding. However, long term clinical studies are required to determine the success rate of the different bulkfillflowable composite resins.

References

[1]. Scotti N, Comba A, Gambino A, Paolino DS, Alovisi M, Pasqualini D, Berutti E. Microleakage at enamel and dentin margins with a bulk fills flowable resin. Eur J Dent 2014; 8: 1-8.

[2]. Sadeghi M. An in vitro microleakage study of class V cavities restored with a new self-adhesive flowable composite resin versus different flowable materials. Dent Res J (Isfahan) 2012; 9(4): 460-465.

[3]. Moorthy A, Hogg CH, Dowling AH, Grufferty BF, Benetti AR, Fleming GJP. Cuspal deflection and microleakage in premolar teeth restored with bulk-fill flowable resin-based composite base materials. J Dent 2012; 40: 500-505.

[4]. Eunice C, Margarida A, João C, Filomena B, Anabela P, Pedro A, Miguel MC, Diana R, Joana M, Mário P, Marques FM. 99mTc in the evaluation of microleakage of composite resin restorations with SonicFill ${ }^{\mathrm{TM}}$ : An in vitro experimental model. Open J Stomatol 2012; 2: 340-347.

[5]. Alomari QD, Barrieshi-Nusair K, Ali M. Effect of C-factor and LED curing mode on microleakage of Class V resin composite restorations. Eu J Dent 2011; 5(4): 400-408.

[6]. Raju RK, Vishwanath BT, Shivanna V. Comparative microleakage evaluation of a flowable composite versus an injectable glass ionomer cement in class II slot preparation-an in vitro study. J Conserv Dent 2003; 6(2): 65-70.

[7]. Puckett A, Fitchie J, Smith J. The effect of incremental versus bulk fill techniques on the microleakage of composite resin using a glass-ionomer liner. Oper Dent 1992; 17(5): 186-191.

[8]. Alberto RF, de Ataide Id. Effect of cyclical lateral forces on microleakage of cervical resin composite restorations. J Conserv Dent 2007; $10: 5-13$

[9]. Ilie N, Bucuta S, Draenert M. Bulk-fill resin-based composites: an in vitro assessment of their mechanical performance.Oper Dent 2013; 38(6): 618-625.

[10]. Van Ende. Bulk-filling of high C-factor posterior cavities: effect on adhesion to cavity-bottom dentin. Dent Mater 2013; 29(3): 269277.

[11]. Lokhande NA, Padmai AS, Sharma U. Effectiveness of Flowable Resin Composite in Reducing Microleakage - An In Vitro Study. J Int Oral Health 2014; 6(3): 111-114.

[12]. Garcia D, Yaman P, Dennison J, Neiva G. Polymerization shrinkage and depth of cure of bulkfillflowable composite resins. Oper Dent 2014;39(4): 441-448.

[13]. Leprince JG, Palin WM, Vanacker J, Sabbagh J, Devaux J, Leloup G. Physico-mechanical characteristics of commercially available bulk-fillcomposites. J Dent 2014; 42(8): 993-1000.

[14]. Francis A.Cuspalflexure and extent of cure of a bulk-fill flowable base composite. Oper Dent 2015;40(5):515-523.

[15]. Agarwal RS, Hiremath H, Agarwal J, Garg A. Evaluation of cervical marginal and internal adaptation using newer bulk fill composites: An in vitro study. J Conserv Dent 2015; 18: 56-61.

[16]. Orłowski M, Tarczydło B, ChałasR.Evaluation of marginal integrity of four bulk-fill dental composite materials: in vitro study. Sci World J 2015; 1: 1-16.

[17]. Jang JH, Park SH, Hwang IN. Polymerization shrinkage and depth of cure of bulk-fill resin composites and highly filled flowableresin.Oper Dent 2015; 40(2): 172-180.

[18]. Kim RJ, Kim YJ, Choi NS, Lee IB. Polymerization shrinkage, modulus, and shrinkage stress related to tooth-restoration interfacial debonding in bulk-fillcomposites. J Dent 2015; 43(4): 430-439.

[19]. Alshali RZ, Salim NA, Satterthwaite JD, Silikas N. Post-irradiation hardness development, chemical softening, and thermal stability of bulk-fill and conventional resin-composites. J Dent 2015; 43(2): 209-218.

[20]. Vandewalker JP, Casey JA, Lincoln TA, Vandewalle KS. Properties of dual-cure, bulk-fill composite resin restorative materials. J Appl Oral Sci2004; 12(1): 56-61. 